\title{
sciendo
}

Int. J. of Applied Mechanics and Engineering, 2019, vol.24, No.1, pp.37-51

DOI: $10.2478 /$ ijame-2019-0002

\section{CHAOTIC CONVECTION OF VISCOELASTIC FLUID IN POROUS MEDIUM UNDER G-JITTER}

\author{
B.S. BHADAURIA* \\ Department of Mathematics, School of Physical and Decisions Sciences \\ Babasaheb Bhimrao Ambedkar University, Lucknow-226025, INDIA \\ E-mail: mathsbsb@yahoo.com
}

\author{
A. SINGH \\ Department of Humanities and Applied Sciences, School of Management Sciences \\ Gosainganj, Lucknow-226501, INDIA \\ E-mail: ajaysingh0044@gmail.com \\ M.K. SINGH \\ Department of Mathematics and Statistics, Banasthli Vidyapith \\ Rajasthan- 304022, INDIA \\ E-mail: s.manojbbau@gmail.com
}

\begin{abstract}
The present article aims at investigating the effect of gravity modulation on chaotic convection of a viscoelastic fluid in porous media. For this, the problem is reduced into Lorenz system (non-autonomous) by employing the truncated Galerkin expansion method. The system shows transitions from periodic to chaotic behavior on increasing the scaled Rayleigh number R. The amplitude of modulation advances the chaotic nature in the system while the frequency of modulation has a tendency to delay the chaotic behavior which is in good agreement with the results due to [1]. The behavior of the scaled relaxation and retardation parameter on the system is also studied. The phase portrait and time domain diagrams of the Lorenz system for suitable parameter values have been used to analyze the system.
\end{abstract}

Key words: non-linear theory; gravity modulation; chaotic convection.

\section{Introduction}

The stability of a convective system depends upon the temperature difference. When the temperature difference in the fluid layer is large enough, the instability occurs in the system due to the buoyant force. This instability makes the system unstable and generates an interesting phenomenon known as convection. The Rayleigh-Bénard convection in a porous medium is called Darcy-Bénard convection or Horton-Rogers problem. Porous media have attracted many researchers during the last three decades due to applications in various fields such as petroleum industry, chemical engineering and geophysics, etc. For more details on the studies of porous media, one can refer to the most excellent books by Ingham and Pop [2], Nield and Bejan [3] and Vafai [4], etc.

In the present model, we consider gravity as a function of time (vertical time-periodic vibrations) known in the literature as gravity modulation or G-jitter which is collinear with actual gravity. This gives two parameters $(\delta, \Omega)$ to control the convective mechanism of the system. Therefore modulation (thermal, gravity, magnetic field) is quite an interesting area for research due to its controlling nature of convective system. Gresho and Sani [5] were the first who proposed the gravity modulated system. In this model they observed that gravity modulation enables the system to get control of its instability either by suitably

\footnotetext{
* To whom correspondence should be addressed
} 
adjusting the values of frequency or the amplitude of modulation. For more details on gravity modulation, see $[6-11]$.

The study of Darcy-Bénard convection with viscoelastic fluid layer is more applicable than Newtonian fluid layer due to its growing importance in various fields such as petroleum, chemical, and reservoir engineering, etc. Industrial fluids are basically non-Newtonian fluids. First of all, Green studied the oscillatory convection in a viscoelastic fluid layer [12], the occurrence of overstability for typical Rayleigh-Bénard convection of a horizontal homogeneous Maxwellian fluid layer heated from below is reported by Vest and Arpaci [13]. Thermal instability in a rotating viscoelastic fluid layer was studied by Bhatia and Steiner [14]. In a horizontal porous layer based on the modified Darcy Oldroyd model, the critical conditions of non-oscillatory instability and oscillatory instability of a viscoelastic fluid were investigated by Kim et al. [15]. Malashetty and Kulkarni [16] used a two field model in the energy equation to investigate the thermal non-equilibrium problem of a Maxwell fluid in a porous medium. Linear and nonlinear analyses on the double-diffusive convection with the Soret effect in a Maxwell fluid-saturated porous medium were made by Wang and Tan [17]. A number of articles based on an oscillatory mode of convection were written by Bhadauria and Kiran [18-20]. These authors derived the complex Ginzburg-Landau equation for evaluating finite amplitude convection and in their model they derived the conditions which confirm the oscillatory flows.

Now-a-days many researchers have taken interest in the study of chaotic convection. Such type of convection is applicable in various kinds of fields, for instance, in the production of crystals and weather sciences, etc. The chaos model was proposed first of all by Poincaré [21]. In this model, the author found that the dynamical system generated by the three body problem is quite sensitive to the initial conditions exhibiting chaotic behavior. Later on, Lorenz [22] studied the system of three ordinary differential equations and developed the model for atmospheric convection; similar work has been done in [23]. Vadasz with their colleague presented a number of articles on the transition to chaotic behavior in a porous layer heated from below [24-28]. Sheu et al. [31] studied chaotic convection of a viscoelastic fluid in a porous medium. Recently, Vadasz [29], Bhadauria and Kiran [1,30] studied chaotic convection in a porous medium by using different physical models.

Thus in the present article, chaotic convection of a viscoelastic fluid in a porous medium under gravity modulation is studied. This type of study helps to handle any kind of convective systems by using appropriate values of modulation parameters. First of all, the adopted model is reduced into a Lorenz system by employing the truncated Galerkin expansion method. The effects of convective parameters which are involved are studied numerically through phase portrait and time domain analyses.

\section{Mathematical structure of the problem}

In this section, we consider an infinitely extended horizontal viscoelastic fluid layer of a porous medium depth $d$, confined between two parallel planes at $z=0$ (lower plane) and $z=d$ (upper plane), which is heated from below as depicted in Fig.1. The Darcy law and Boussinesq approximation are used to solve the model equations. The governing equations of this model are [31]

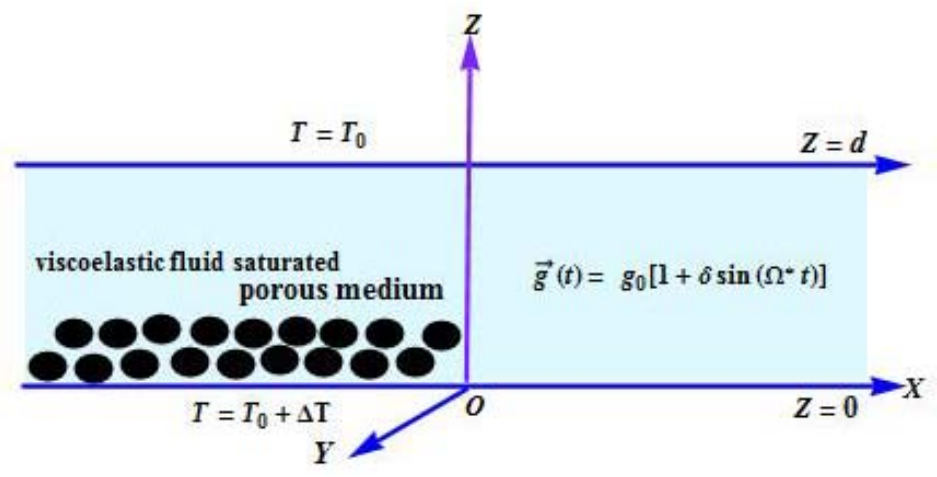

Fig.1. Physical configuration of the problem. 


$$
\nabla \cdot \boldsymbol{q}=0
$$

$$
\begin{aligned}
& \left(\bar{\lambda}_{1} \frac{\partial}{\partial t}+1\right)\left(\frac{\rho_{0}}{\phi} \frac{\partial q}{\partial t}+\nabla p-\rho \boldsymbol{g}\right)+\frac{\mu}{K}\left(\bar{\lambda}_{2} \frac{\partial}{\partial t}+1\right) \boldsymbol{q}=0, \\
& \frac{\partial T}{\partial t}+(\boldsymbol{q} \cdot \nabla) T=\kappa_{T} \nabla^{2} T, \\
& \rho=\rho_{0}\left\{1-\alpha_{T}\left(T-T_{0}\right)\right\},
\end{aligned}
$$

where the physical variables have their usual meanings as given in the nomenclature. The externally imposed gravitational field and the thermal boundary conditions are given by

$$
\begin{aligned}
& \boldsymbol{g}=g_{0}\left[1+\delta \sin \left(\Omega^{*} t\right)\right] \hat{k}, \\
& T=\left\{\begin{array}{ccc}
T_{0}+\Delta T ; & \text { if } & z=0, \\
T_{0} ; & \text { if } & z=d,
\end{array}\right.
\end{aligned}
$$

where $g_{0}$ is the mean gravity and $\hat{k}$ is the unit vector along the positive $z$-axis.

\section{Basic state}

In the basic state the velocity and temperature profiles are given by

$$
q_{b}=0, \quad p=p_{b}(z), \quad T=T_{b}(z), \quad \rho=\rho_{b}(z) .
$$

Using Eq.(3.1) in Eqs (2.1), the following relations are obtained

$$
\begin{aligned}
& \frac{\partial p_{b}}{\partial z}=-\rho_{b} \boldsymbol{g}, \\
& \kappa_{T} \frac{d^{2} T}{d z^{2}}=0, \\
& \rho_{b}=\rho_{0}\left\{1-\alpha_{T}\left(T_{b}-T_{0}\right)\right\} .
\end{aligned}
$$

The dimensionless solution of Eq.(3.3) subject to the boundary conditions (2.3) is given by

$$
T_{b}=1-z
$$

Now, we superimpose finite amplitude perturbations on the basic state in the form

$$
\boldsymbol{q}=q_{b}+q^{\prime}, \quad T=T_{b}+T^{\prime}, \quad p=p_{b}+p^{\prime}, \quad \rho=\rho_{b}+\rho^{\prime},
$$


where the primes represent the perturbed quantities. According to Sheu et al. [31] and Bhadauria and Kiran [30] the dimensionless governing system is given by

$$
\begin{aligned}
& \left(\frac{1}{\operatorname{Pr} \mathrm{D}}\left(\lambda_{1} \frac{\partial}{\partial t}+1\right) \frac{\partial}{\partial t}+\left(\lambda_{2} \frac{\partial}{\partial t}+1\right)\right) \nabla^{2} \psi=g_{m} \operatorname{RaD}\left(\lambda_{1} \frac{\partial}{\partial t}+1\right) \frac{\partial T}{\partial x} \\
& -\frac{\partial \psi}{\partial x} \frac{\partial T_{b}}{\partial z}+\left(\frac{\partial}{\partial t}-\nabla^{2}-R_{i}\right) T=\frac{\partial(\psi, T)}{\partial(x, z)}
\end{aligned}
$$

where $\operatorname{PrD}$ is the Darcy-Prandtl number, $\mathrm{Da}=\frac{K}{d^{2}}$ is the Darcy number, $\operatorname{Pr}=\frac{v}{k_{T}}$ is the Prandtl number, $\mathrm{RaD}=\frac{\alpha_{T} g_{0} \Delta T K d}{\nu \kappa_{T}}$ is the Darcy Rayleigh number, $\nu=\frac{\mu}{\rho_{0}}$ is the kinematic viscosity and $g_{m}=\left(1+\delta \sin \left(\Omega^{*} t\right)\right)$ conditions

The above dimensionless system will be solved by considering stress free and isothermal boundary

$$
\psi=\frac{\partial^{2} \psi}{\partial z^{2}}=T=0 \quad \text { on } \quad z=0, \quad z=1
$$

\section{Method of solution}

The solution of nonlinear Eqs (3.7) and (3.8) subject to the boundary conditions (3.9) is obtained by using the truncated Galerkin expansion method. The stream function and temperature field are taken in the forms as mentioned in [29]

$$
\begin{aligned}
& \psi=A_{11} \sin \left(\frac{\pi x}{L}\right) \sin (\pi z) \\
& T=T_{b}+B_{11} \cos \left(\frac{\pi x}{L}\right) \sin (\pi \mathrm{z})+B_{02} \sin (2 \pi \mathrm{z})
\end{aligned}
$$

Using Eqs (4.1) and (4.2) into Eqs (3.7) and (3.8), multiplying the equations by orthogonal eigenfunctions corresponding to Eqs (4.1) and (4.2), and then integrating them over the spatial domain, yield a set of three differential equations for the time evolution of the amplitudes, in the form of

$$
\begin{aligned}
& {\left[\left(1+\Gamma \frac{d}{d \tau}\right) \frac{d}{d \tau}+\frac{\operatorname{Pr} \mathrm{D} \gamma}{\pi^{2}}\left(1+\Gamma \Lambda \frac{d}{d \tau}\right)\right] A_{11}=\frac{\operatorname{Pr} \mathrm{D} \gamma R_{a D} g_{m}}{\chi \pi^{3}}\left(1+\Gamma \frac{d}{d \tau}\right) B_{11},} \\
& \frac{d B_{11}}{d \tau}=-B_{11}+\frac{1}{\pi \chi} A_{11}+\frac{A_{11} B_{02}}{\chi}, \\
& \frac{d B_{02}}{d \tau}=-\frac{1}{2 \chi} A_{11} B_{11}-4 \gamma B_{02},
\end{aligned}
$$


where the time has been re-scaled and the following notations are introduced

$$
\tau=\frac{\left(L^{2}+1\right) \pi^{2}}{L^{2}} t, \quad \chi=\frac{L^{2}+1}{L}, \quad \gamma=\frac{L^{2}}{L^{2}+1}, \quad \Omega=\frac{L^{2}}{\left(L^{2}+1\right) \pi^{2}} \Omega^{*}, \quad \sigma=\frac{\operatorname{Pr} \mathrm{D} \gamma}{\pi^{2}} \quad \text { and } \quad \mathrm{R}=\frac{\operatorname{RaD}}{\pi^{2} \chi^{2}} \text {, }
$$

again rescale the amplitude in the form of

$$
X=\frac{A_{11}}{2 \chi \sqrt{2 \gamma(R-1)}}, \quad Y=\frac{\pi R B_{11}}{2 \sqrt{2 \gamma(R-1)}}, \quad Z=-\frac{\pi R B_{02}}{(R-1)}
$$

which provide the following set of equations

$$
\begin{aligned}
& \frac{d X}{d \tau}=W \\
& \frac{d Y}{d \tau}=R X-Y-(R-1) X Z, \\
& \frac{d Z}{d \tau}=4 \gamma(X Y-Z), \\
& \frac{d W}{d \tau}=\sigma\left[\left(R g_{m}-\frac{1}{\Gamma}\right) X+\left(\frac{1}{\Gamma}-1\right) g_{m} Y-(R-1) g_{m} X Z-\left(\Lambda+\frac{1}{\sigma \Gamma}\right) W\right]
\end{aligned}
$$

where $g_{m}$ is the function of rescaled time $\tau$. If the amplitude of gravity modulation $\delta=0$, then the system (4.6) reduces into Sheu et al. [31] model.

\section{Results and discussion}

MATHEMATICA 7.0 has been used for the numerical simulation of the Lorenz system (4.6). In this simulation, the initial conditions are $\tau=0: X=Y=Z=0.9, W=0.1$ and the parameters $\sigma=10, \gamma=0.5$ are fixed. The parameters $\mathrm{R}, \delta, \Omega, \Gamma, \Lambda$ are considered as variable to examine the effect of gravity modulation on the chaotic system. It is assumed that the amplitude of gravity modulation $\delta$ is very small. The phase-portrait diagrams depict how modulation terms affect the dynamics of the thermal convection for a combination of varied parameters. Vadasz et al. [29] studied the effect of gravity modulation on chaotic convection for variable frequency $\Omega$ with constant amplitude $\delta$, while in this paper, these effects are studied for variable frequency $\Omega$ and amplitude $\delta$. The results are further depicted in Figs 2-7 to analyze the Lorenz model by using phase-portrait and time domain diagrams.

The effect of the scaled Rayleigh number $\mathrm{R}$ on the system is depicted in Figs $2 \mathrm{a}-2 \mathrm{~d}$, keeping fixed the other parameters. Figures $2 \mathrm{a}(\mathrm{R}=5)$ and $2 \mathrm{~b}(\mathrm{R}=10)$ show a periodic solution but this periodicity of the system is not long lasting. Figure $2 \mathrm{c}(\mathrm{R}=12)$ depicts a chaotic behavior or a periodic solution of the Lorenz system, which is due to the fact that heat transfer is larger on increasing $\mathrm{R}$ as is clear from the figures. Therefore, in case of gravity modulation the system shows periodic solution whenever $\mathrm{R}<12$ (appox.) and show always chaotic behavior when $\mathrm{R} \geq 12$, given in Fig.2d $(\mathrm{R}=75)$ compatible with the result of [31]. 

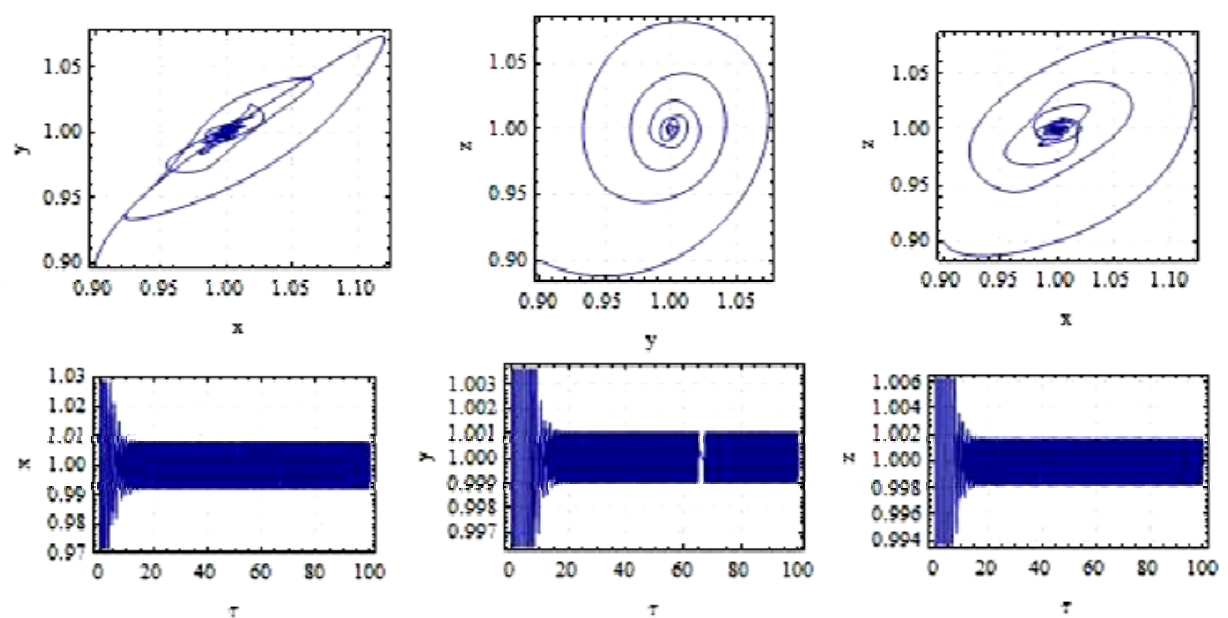

Fig.2a. Phase portrait and time domain diagrams for the system (4.6) with parameters $\Gamma=1, \Lambda=0.7, \mathrm{R}=5, \delta=0.1, \Omega=10$.
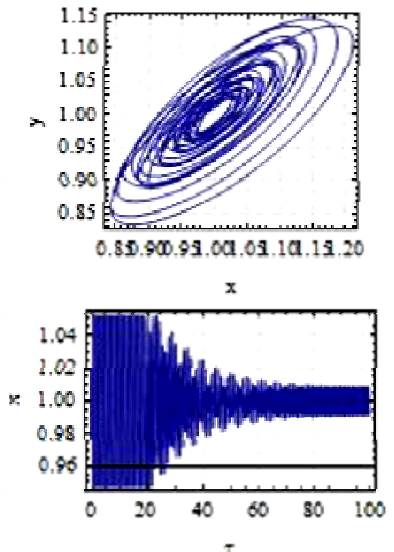

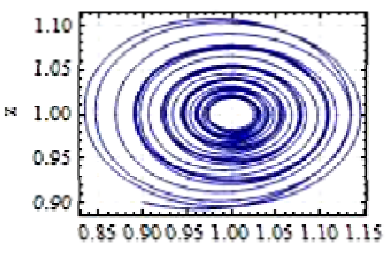

y

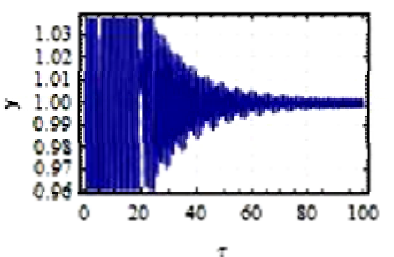

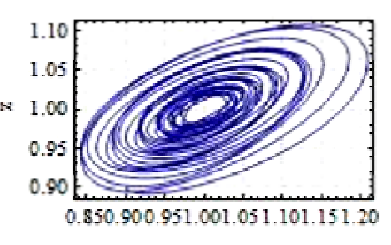

$\mathbf{x}$

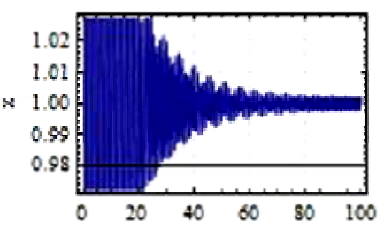

Fig.2b. Phase portrait and time domain diagrams for the system (4.6) with parameters $\Gamma=1, \Lambda=0.7, \mathrm{R}=10, \delta=0.1, \Omega=10$.
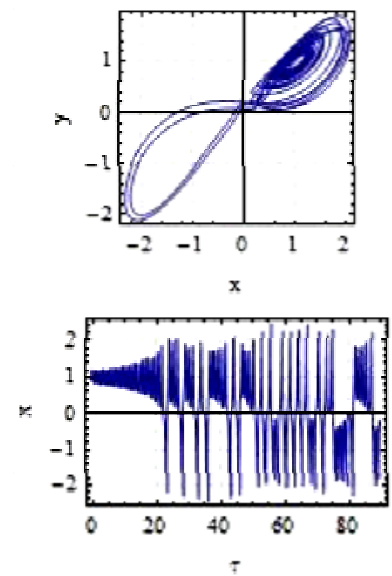
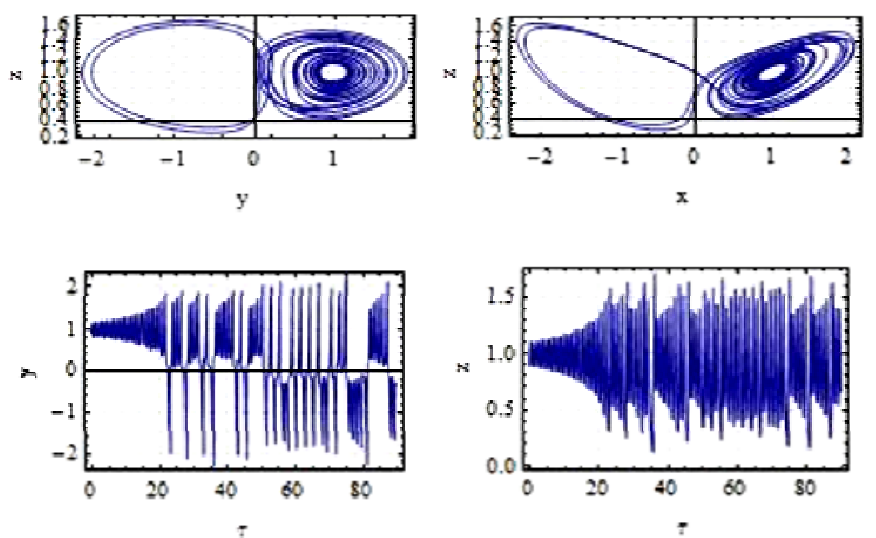

Fig.2c. Phase portrait and time domain diagrams for the system (4.6) with parameters $\Gamma=1, \Lambda=0.7, \mathrm{R}=12, \delta=0.1, \Omega=10$. 

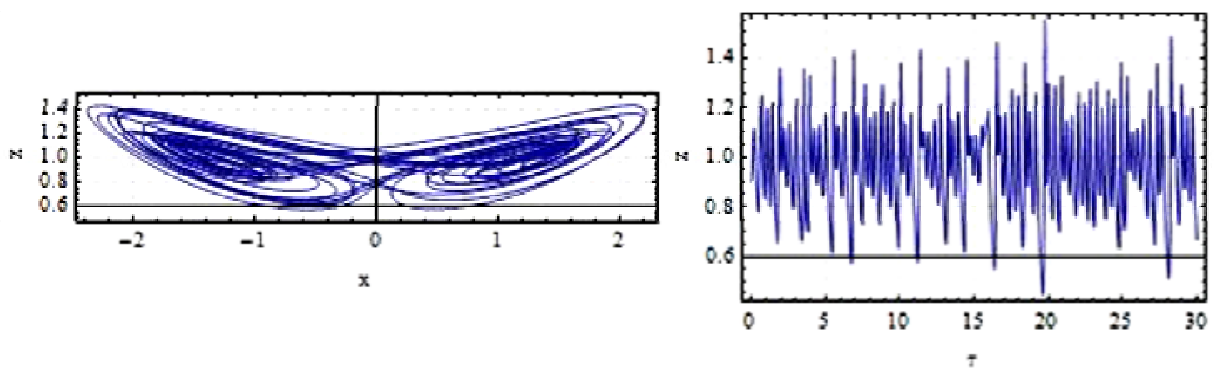

Fig.2d. Phase portrait time domain diagrams for the system (4.6) with parameters $\Gamma=1, \Lambda=0.7, \mathrm{R}=75, \delta=0.1, \Omega=10$.
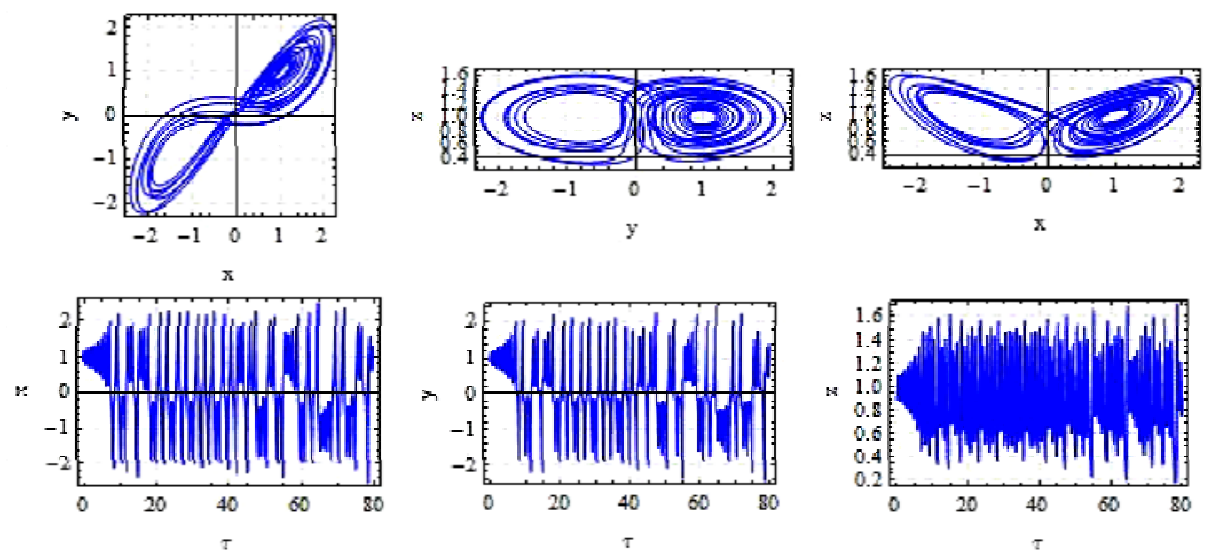

Fig.3a. Phase portrait and time domain diagrams for the system (4.6) with parameters $\Gamma=1, \Lambda=0.7, \mathrm{R}=15, \delta=0.01, \Omega=10$.

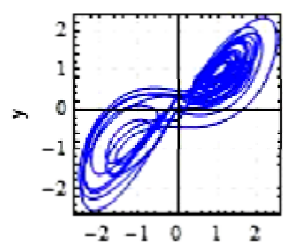

$\mathrm{x}$

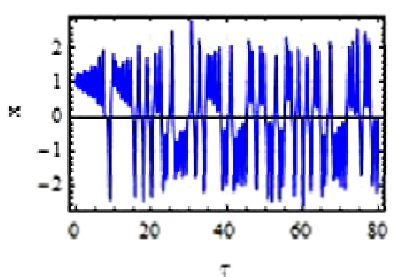

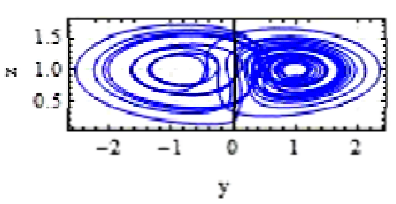
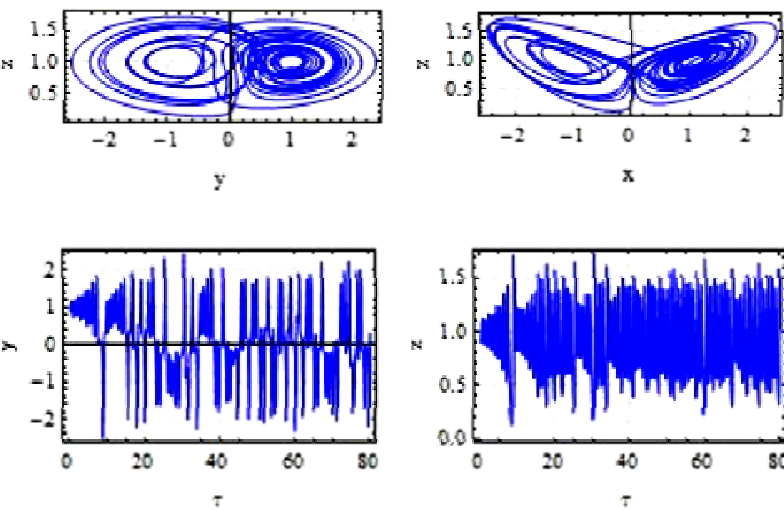

Fig.3b. Phase portrait and time domain diagrams for the system (4.6) with parameters $\Gamma=1, \Lambda=0.7, \mathrm{R}=15, \delta=0.2, \Omega=10$. 


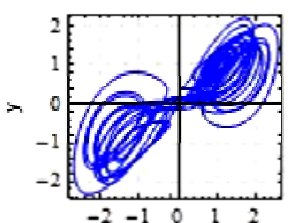

$\mathrm{x}$

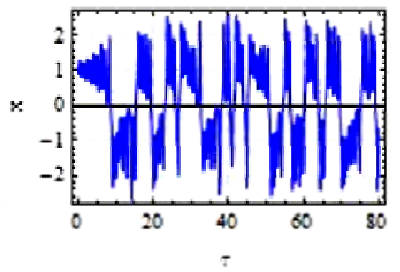

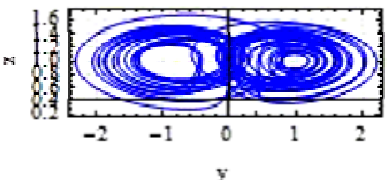
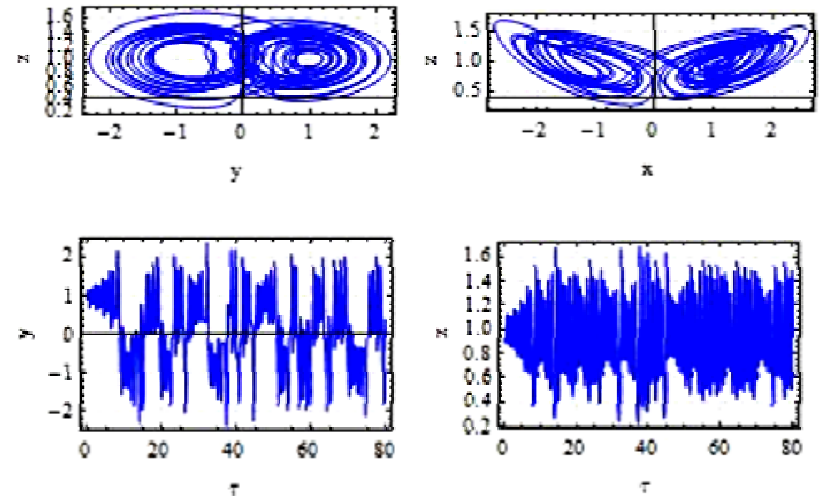

Fig.3c. Phase portrait and time domain diagrams for the system (4.6) with parameters $\Gamma=1, \Lambda=0.7, \mathrm{R}=15, \delta=0.3, \Omega=10$.

The impact of amplitude of gravity modulation $\delta$ on the system for different parametric values $\delta$ $=0.01,0.2,0.3$, keeping fixed other parameters, is depicted in Figs 3a, 3b, 3c, respectively. These figures show that the trajectories are much disturbed on increasing $\delta$. Therefore, the chaotic behavior advances, that is, the heat transfer increases gradually [1]. Figures $4 a, 4 b, 4 c$ depict the effect of different frequencies of gravity modulation $\Omega=10,100,200$, keeping fixed the other parameters. The phase-portrait diagrams and time domain solutions show that the system has a chaotic nature for $\Omega=10$ while the system shows a periodic nature for $\Omega=100,200$. Thus, the system returns to periodic solution from the chaotic solution as $\Omega$ increases, and so, frequency of modulation $\Omega$ delays the heat transfer [1].

The effect of the scaled relaxation parameter $\Gamma$ can be seen in Figs $5 a, 5 b, 5 c$ for its different values $\Gamma=0.3,0.35,0.45$, keeping fixed the other parameters, respectively. It is evident that on increasing $\Gamma$ the disturbance in the solution the increases, which is depicted by phase-portrait and time domain diagrams. The system loses its periodicity and transfers into a chaotic solution, and so, heat transfer advances convection [1]. The scaled retardation parameter $\Lambda$ shows an opposite effect of the scaled relaxation parameter $\Gamma$, and is depicted in Figs $6 \mathrm{a}, 6 \mathrm{~b}, 6 \mathrm{c}$ for $\Lambda=0.5,0.7,0.9$, keeping fixed other parameters. In this case, the system loses its chaotic behavior and shows a periodic behavior, and so, heat transfer delays convection [1].
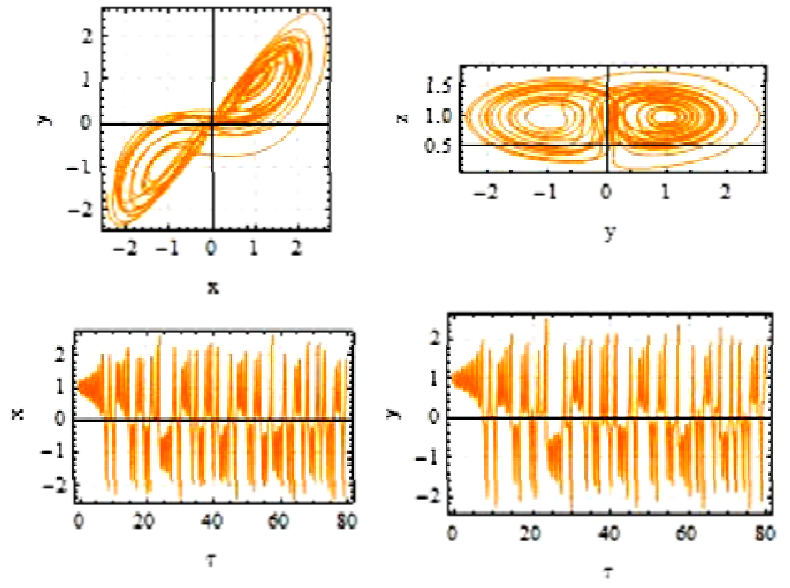
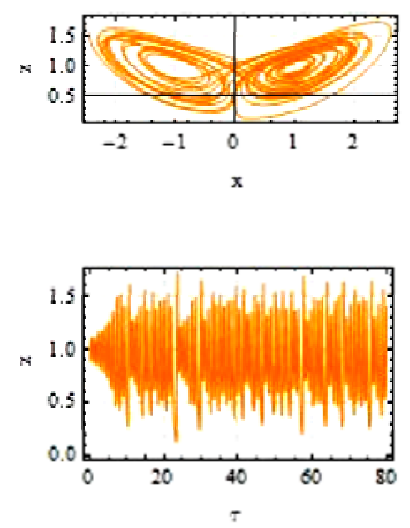

Fig.4a.Phase portrait and time domain diagrams for the system (4.6) with parameters $\Gamma=1, \Lambda=0.7, \mathrm{R}=15, \delta=0.1, \Omega=10$. 

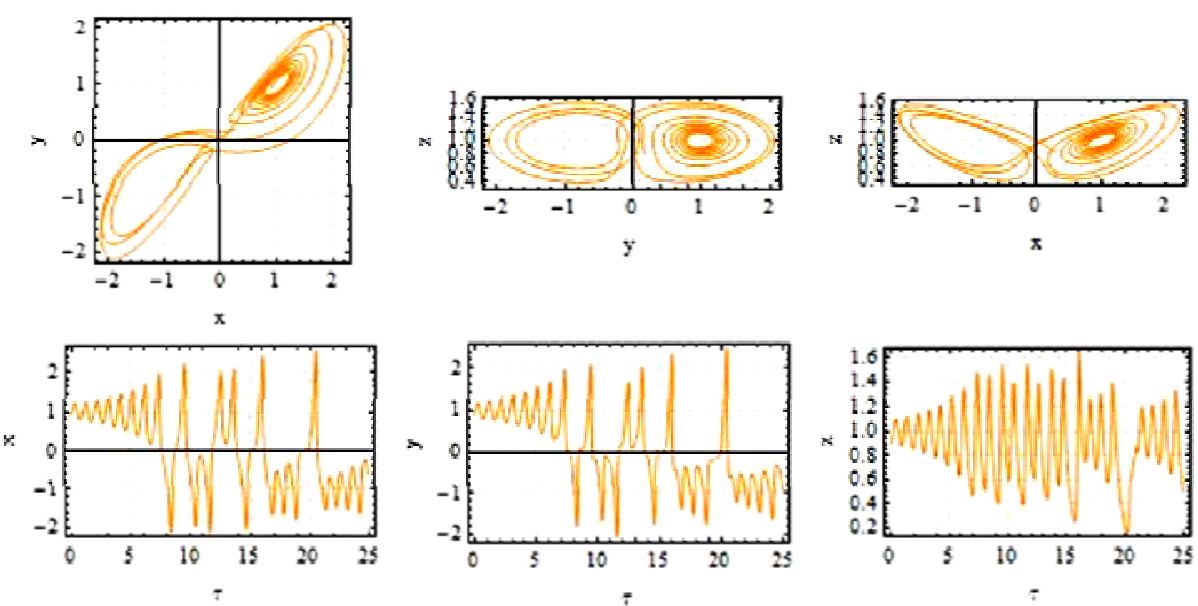

Fig.4b. Phase portrait and time domain diagrams for the system (4.6) with parameters $\Gamma=1, \Lambda=0.7, \mathrm{R}=15, \delta=0.1, \Omega=100$.
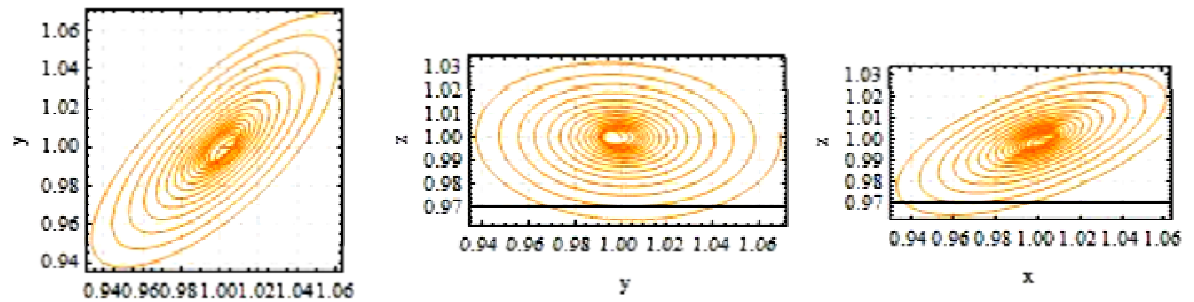

$\mathrm{x}$
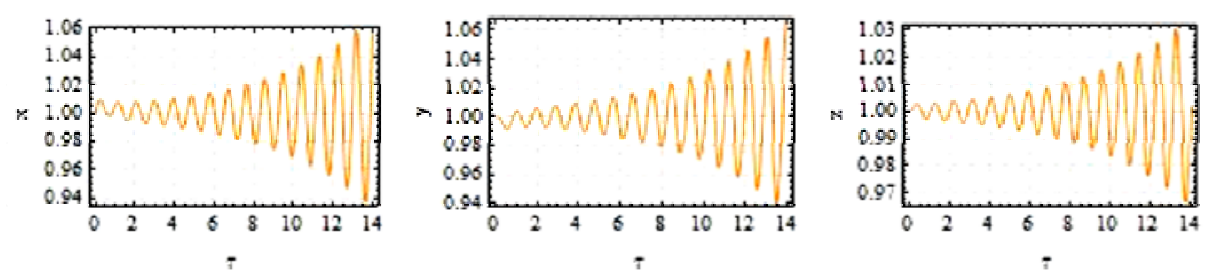

Fig.4c. Phase portrait and time domain diagrams for the system (4.6) with parameters $\Gamma=1, \Lambda=0.7, \mathrm{R}=15, \delta=0.1, \Omega=200$.
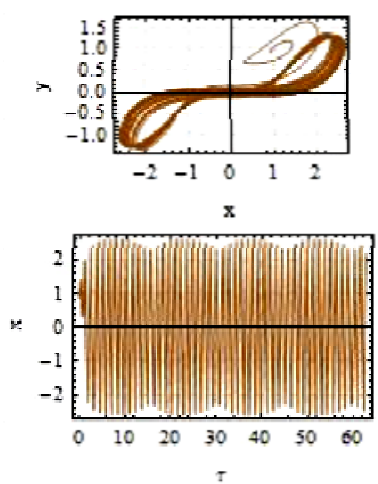

$\therefore$
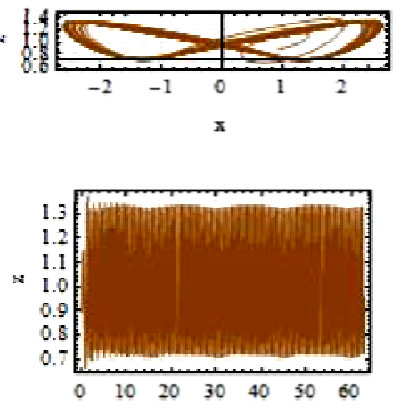

Fig.5a. Phase portrait and time domain diagrams for the system (4.6) with parameters $\Gamma=0.3, \Lambda=0.1, \mathrm{R}=5, \delta=0.1, \Omega=10$. 


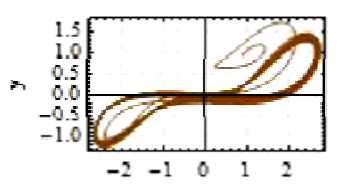

$\mathrm{x}$

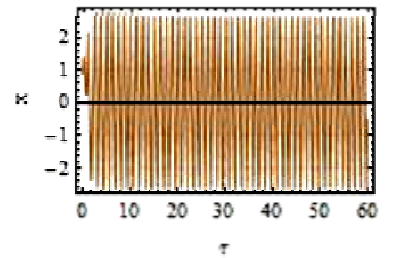

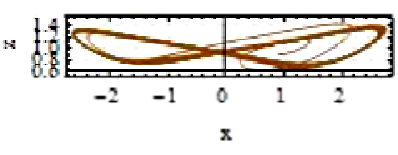

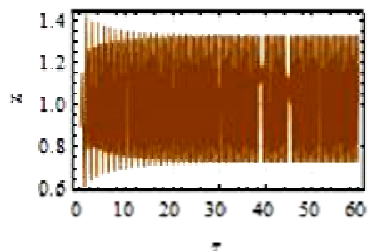

Fig.5b. Phase portrait and time domain diagrams for the system (4.6) with parameters $\Gamma=0.35, \Lambda=0.1, \mathrm{R}=5, \delta=0.1, \Omega=10$.
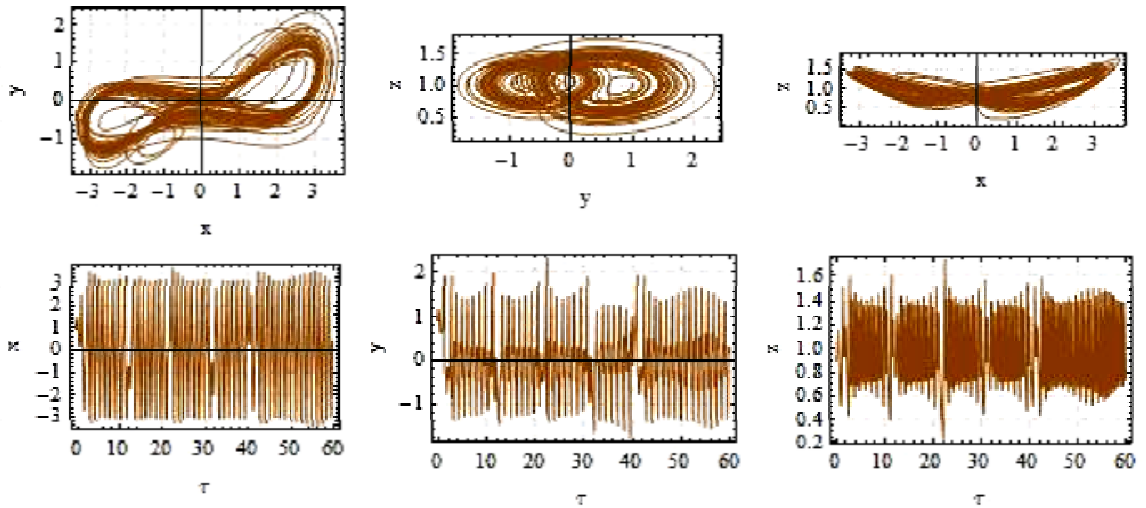

Fig.5c. Phase portrait and time domain diagrams for the system (4.6) with parameters $\Gamma=0.45, \Lambda=0.1, \mathrm{R}=5, \delta=0.1, \Omega=10$.

Finally, we compare the result of gravity modulated and unmodulated systems. For $\delta=0, R=1.1$, Fig.7a shows a stable solution [31] while for $\delta=0.1, \mathrm{R}=1.1$, the system has periodic solution, depicted in Fig. $7 \mathrm{~b}$. We noticed that in the gravity modulated system heat transfer is larger in comparison to the unmodulated system.

\section{Conclusions}

In this paper, chaotic convection of a viscoelastic fluid in a porous medium under gravity modulation is studied. The adopted model is first reduced into a Lorenz system by employing the trauncated Galerkin expansion method. By using phase portrait and time domain diagrams the following findings are obtained

a) The effect of the scaled Rayleigh number $\mathrm{R}$ is to increase the heat transport in the Lorenz system.

b) The amplitude $\delta$ (frequency $\Omega$ ) of modulation is to advance (delay) the heat transfer in the Lorenz system.

c) The scaled relaxation parameter $\Gamma$ has a tendency to advance the chaotic behaviour whereas the scaled retardation parameter $\Lambda$ delays the chaotic behaviour in the system.

d) Finally, it is obtained that heat transfer is larger in the modulated system in comparison to the unmodulated system. 

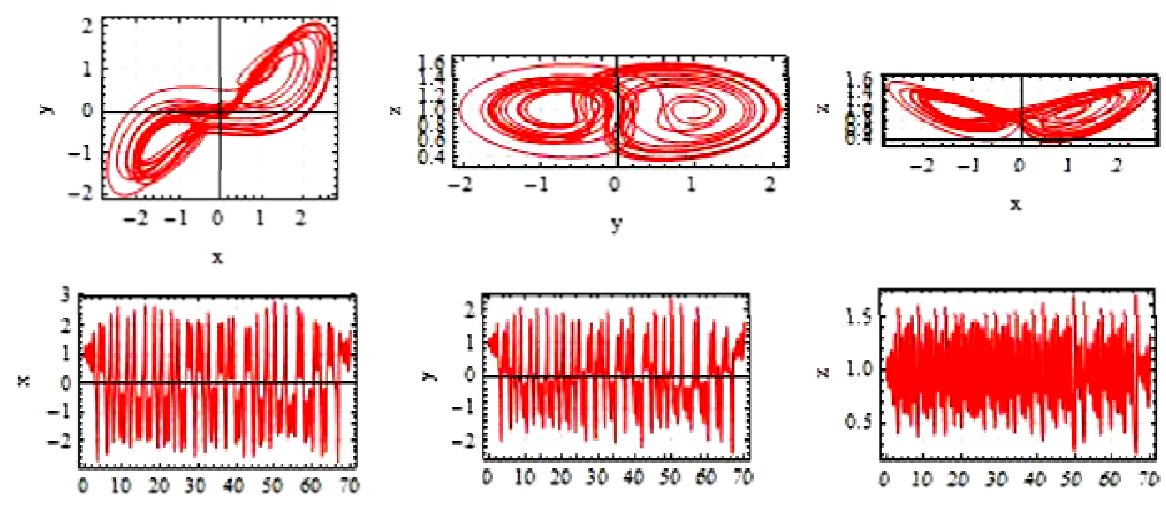

Fig.6a. Phase portrait and time domain diagrams for the system (4.6) with parameters $\Gamma=1, \Lambda=0.5, \mathrm{R}=15, \delta=0.1, \Omega=10$.
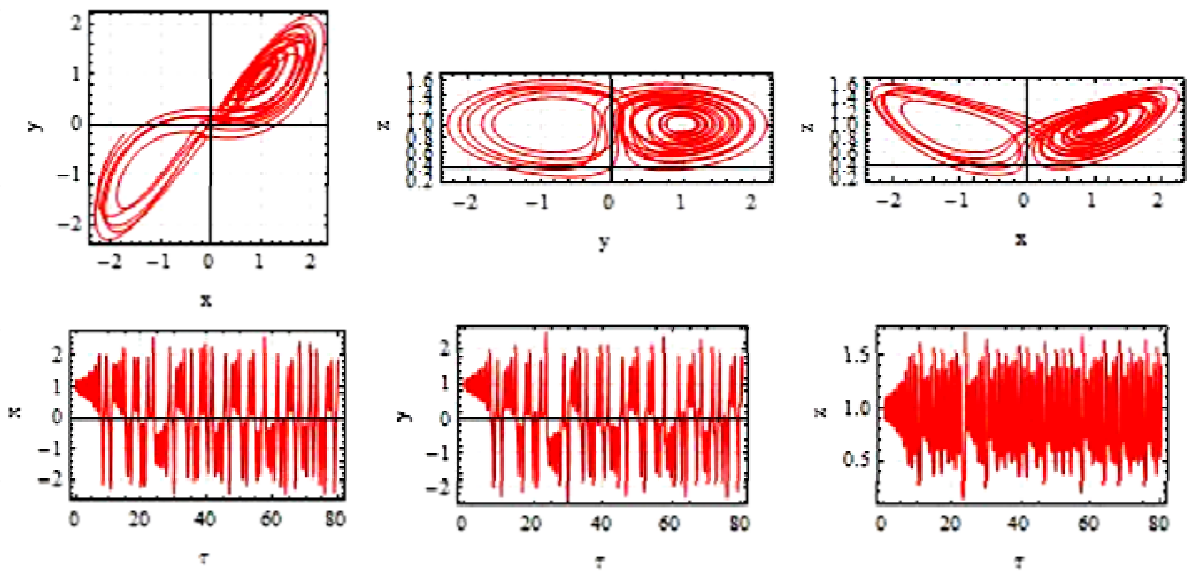

Fig.6b. Phase portrait and time domain diagrams for the system (4.6) with parameters $\Gamma=1, \Lambda=0.7, \mathrm{R}=15, \delta=0.1, \Omega=10$.
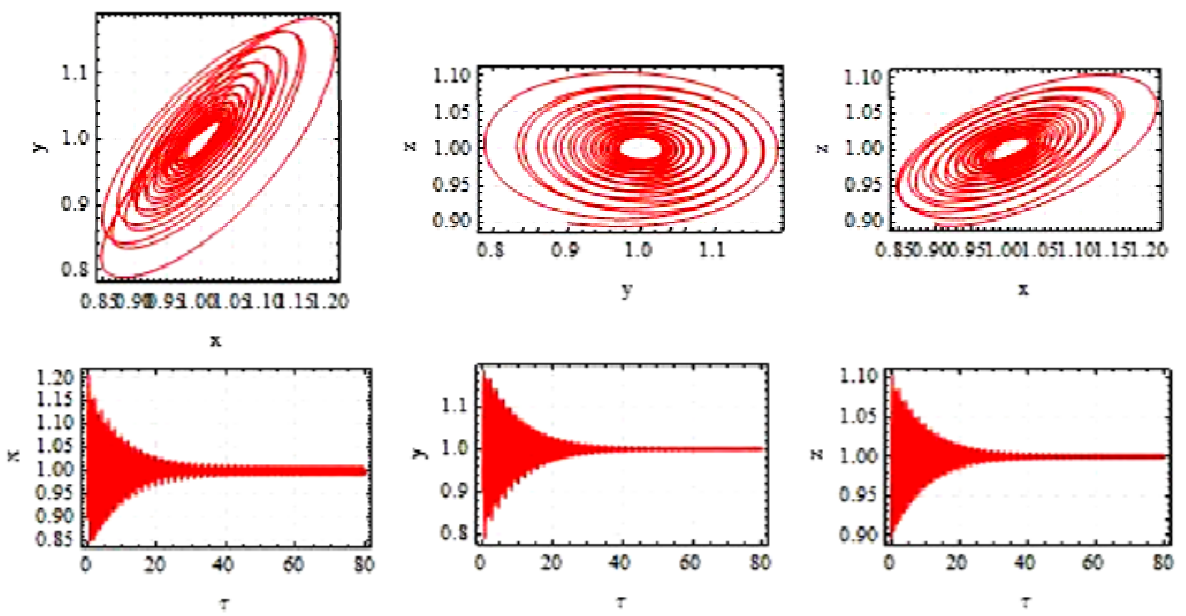

Fig.6c. Phase portrait and time domain diagrams for the system (4.6) with parameters $\Gamma=1, \Lambda=0.9, \mathrm{R}=15, \delta=0.1, \Omega=10$. 

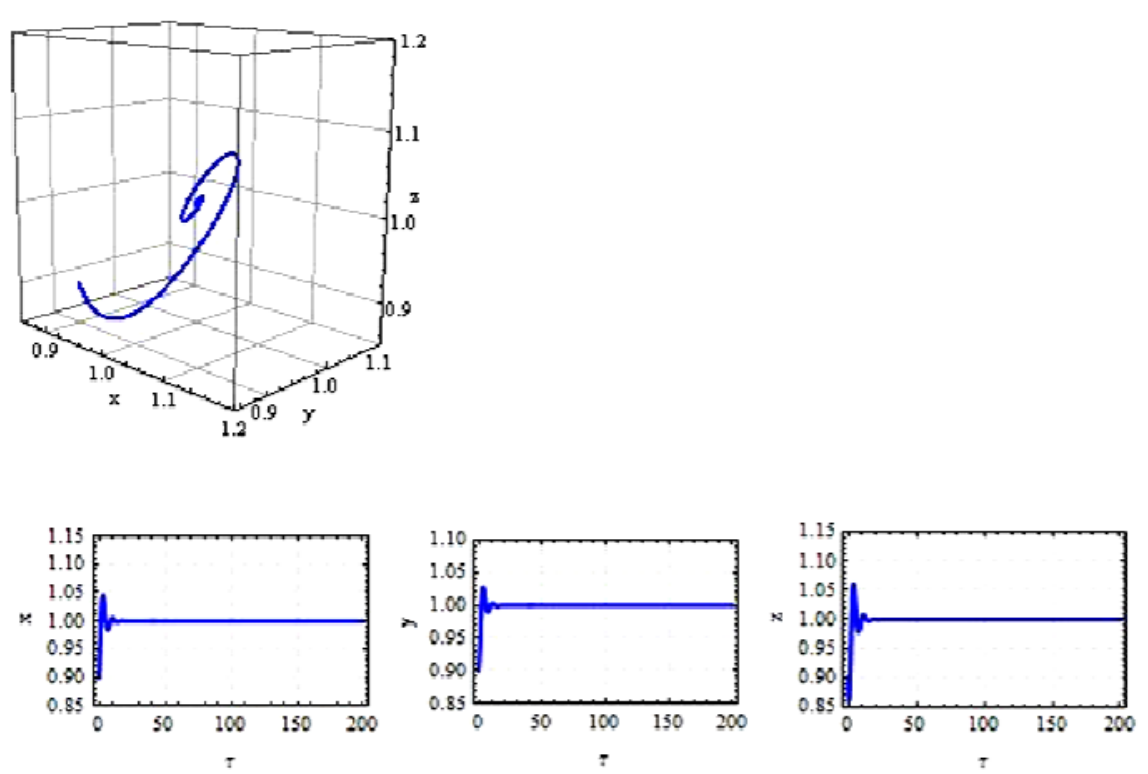

Fig.7a. 3D phase portrait and time domain diagrams for the system (4.6) with parameters $\Gamma=1, \Lambda=0.1, \mathrm{R}=1.1, \delta=0, \Omega=10$.
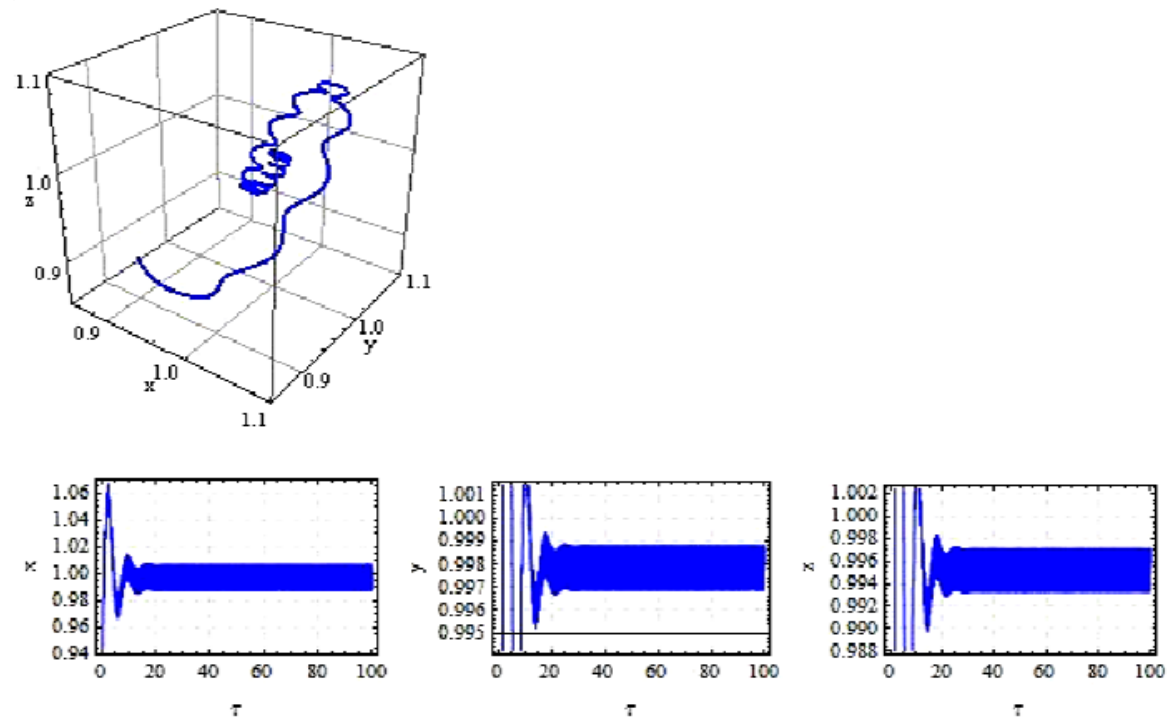

Fig.7b. 3D phase portrait and time domain diagrams for the system (4.6) with parameters $\Gamma=1, \Lambda=0.1, \mathrm{R}=1.1, \delta=0.1, \Omega=10$.

\section{Acknowledgement}

The authors Ajay Singh and Manoj Kumar Singh gratefully acknowledge the financial assistance from BabasahebBhimraoAmbedkar University, Lucknow, India, in the form of a research fellowship. 


\section{Nomenclature}

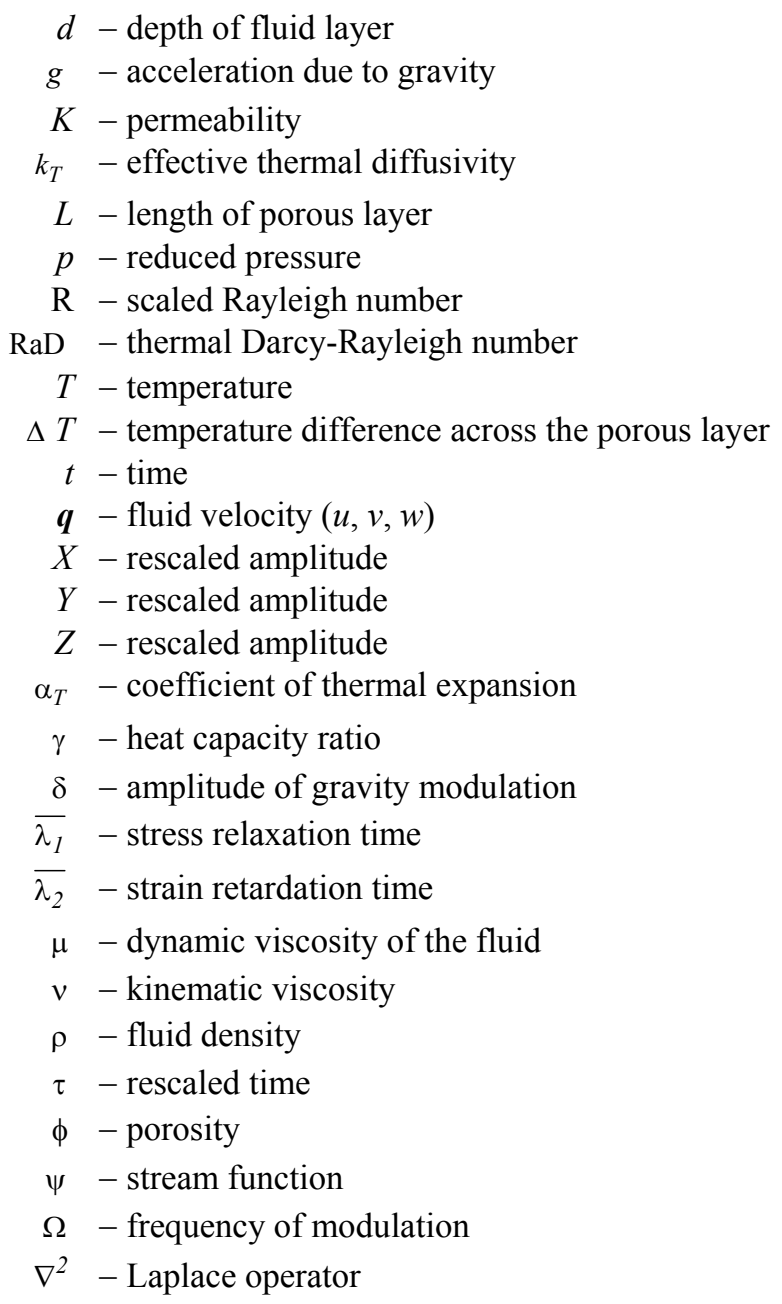

\section{Subscripts}

$B$ - basic state

$O$ - reference value

\section{Superscripts}

' - perturbed quantity

* - dimensionless quantity

\section{References}

[1] Bhadauria B.S. and Kiran P. (2015): Chaotic and oscillatory magneto-convection in a binary viscoelastic fluid under G-jitter. - Int. J. Heat Mass Transf., vol.84, pp.610-624.

[2] Ingham D.B. and Pop I. (2005): Transport Phenomena in Porous Media. - 1st Edn., vol.3, Elsevier, Oxford.

[3] Nield D.A. and Bejan A. (2006): Convection in Porous Media. - 3rd Edn. New York: Springer.

[4] Vafai K. (2000): Handbook of Porous Media. - New York: Marcel Dekker. 
[5] Gresho P.M. and Sani R. (1970): The effects of gravity modulation on the stability of a heated fluid layer. - J. Fluid Mech., vol.40, pp.783-806.

[6] Govender S. (2005): Weak non-linear analysis of convection in a gravity modulated porous layer. - Transp. Porous Media, vol.60, pp.33-42.

[7] Malashetty M.S. and Padmavathi V. (1997): Effect of gravity modulation on the onset of convection in a fluid and porous layer. - Int. J. Engg. Science, vol.35, pp.829-839.

[8] Malashetty M.S. and Swamy M. (2011): Effect of gravity modulation on the onset of thermal convection in rotating fluid and porous layer. - Phys Fluids, vol.23, No.6, pp.064108.

[9] Rees D.A.S. and Pop I. (2000): The effect of G-jitter on vertical free convection boundary-layer flow in porous media. - Int. Comm. Heat Mass Transfer, vol.27, No.3, pp.424.

[10] Siddhavaram V.K. and Homsy G.M. (2006): The effects of gravity modulation on fluid mixing Part 1. Harmonic modulation. - J. Fluid Mech., vol.562, pp.445-475.

[11] Saravanan S. and Sivakumar T. (2011): Thermo vibrational instability in a fluid saturated anisotropic porous medium. - ASME, J. Heat Transfer, vol.133, No.5, 051601, doi:10.1115/1.4003013.

[12] Green T. (1968) III: Oscillating convection in an elasticoviscous liquid. - Phys. Fluids, vol.11, 1410.

[13] Vest C.M. and Arpaci V.S. (1969): Overstability of a viscoelastic fluid layer heated from below. - J. Fluid Mech., vol.36, pp.613-623.

[14] Bhatia P.K. and Steiner J.M. (1972): Convective instability in a rotating viscoelastic fluid layer. - ZAMM 52, pp.321-327.

[15] Kim M.C., Lee S.B., Kim S. and Chung B.J. (2003): Thermal instability of viscoelastic fluids in porous media. Int. J. Heat Mass Transfer, vol.46, pp.5065-5072.

[16] Malashetty M.S. and Kulkarni S. (2009): The convective instability of Maxwell fluid-saturated porous layer using a thermal non-equilibrium model. - J. Non Newton Fluid Mech., vol.162, No.1-3, pp.29-37.

[17] Wang S. and Tan W. (2011): Stability analysis of Soret-driven double-diffusive convection of Maxwell fluid in a porous medium. - Int. J. Heat Fluid Flow, vol.32, No.1, pp.88-94.

[18] Bhadauria B.S. and Kiran P. (2014): Weak non-linear oscillatory convection in a viscoelastic fluid saturated porous medium under gravity modulation. - Transp. Porous Media, vol.104, pp.451-467.

[19] Bhadauria B.S. and Kiran P. (2014): Weak non-linear oscillatory convection in a viscoelastic fluid layer under gravity modulation. - Int. J. Non-Linear Mech., vol.65, pp.133-140.

[20] Bhadauria B.S. and Kiran P. (2014): Weakly non-linear oscillatory convection in a viscoelastic fluid saturating porous medium under temperature modulation. - Int. J. Heat Mass Transfer, vol.77, pp.843-851.

[21] Poincaré J.H. (1890): Sur le probléme des trois corps et les équations de la dynamique. - Acta Mathematica, vol.13, pp.01-279.

[22] Lorenz E.N. (1963): Deterministic non-periodic flow. - J. Atmos Sci., vol.20, pp.130-141.

[23] Sparrow C. (1982): The Lorenz Equations: Bifurcations, Chaos, and Strange Attractors. - New York: SpringerVerlag.

[24] Vadasz P. and Olek S. (1998): Transition and chaos for free convection in a rotating porous layer. - Int. J. Heat Mass Transfer, vol.41, No.11, pp.1417-1435.

[25] Vadasz P. and Olek S. (1999): Weak turbulence and chaos for low Prandtl number gravity driven convection in porous media. - Transp. Porous Media, vol.37, No.1, pp.69-91.

[26] Vadasz P. and Olek S. (1999): Computational recovery of the homoclinic orbit in porous media convection. - Int. J. Non-Linear Mech., vol.34, No.6, pp.89-93. 
[27] Vadasz P. and Olek S. (2000): Route to chaos for moderate Prandtl number convection in a porous layer heated from below. - Transp. Porous Med., vol.41, No.2, pp.211-239.

[28] Vadasz P. and Olek S. (2000): Convergence and accuracy of Adomian's decomposition method for the solution of Lorenz equations. - Int. J. Heat Mass Transfer, vol.43, No.10, pp.1715-1734.

[29] Vadasz et al. (2014): Chaotic and Periodic natural convection for moderate and high Prandtl numbers in a porous layer subject to vibrations. - Transp. Porous Media, vol.103, pp.279-294.

[30] Bhadauria B.S. and Kiran P. (2015): Chaotic convection in a porous medium under temperature modulation. Transp. Porous Media, vol.107, pp.745-763.

[31] Sheu L.-J., Tam L.-M., Chen J.-H., Chen H.-K., Lin K.-T. and Kang Y. (2008): Chaotic convection of viscoelastic fluid in porous media. - Chaos, Solitons and Fractals, vol.37, No.1, pp.113-124.

Received: August 18, 2017

Revised: July 31, 2018 